\title{
Resolving temporal Gribov copies in Coulomb gauge Yang-Mills theory
}

\author{
H. Reinhardt and P. Watson \\ Institut für Theoretische Physik, Universität Tübingen, \\ Auf der Morgenstelle 14, D-72076 Tübingen, Deutschland
}

\begin{abstract}
The continuum Yang-Mills functional integral within the first order formalism and in Coulomb gauge is studied. In particular, the temporal zero-modes of the Faddeev-Popov operator are explicitly accounted for. It is shown that the treatment of these zero-modes results in the constraint that the total color charge of the system vanishes at all times. Further, it is argued that the functional integral is effectively fully gauge-fixed once Gauss' law has been resolved in Coulomb gauge.
\end{abstract}

PACS numbers: 11.15.Tk,12.38.Aw

\section{INTRODUCTION}

Coulomb gauge quantum chromodynamics $[\mathrm{QCD}]$ and Yang-Mills theory have recently begun to attract considerable attention, primarily because of their great potential in studying confinement. This potential has long been recognized and led to the Gribov-Zwanziger scenario for confinement [1-3] whereby the temporal component of the gluon propagator provides for a long-range confining force whilst the transverse spatial components are suppressed in the infrared. However, progress in Coulomb gauge has been hindered by the inherent noncovariance of the gauge condition. Gratifyingly, the technical obstacles are being steadily overcome.

Various approaches to Coulomb gauge are currently being considered. There exist lattice studies [4-9] that are beginning to shed light on the nonperturbative behavior of the propagators. Also, a Hamiltonian-based approach to the problem [10-13] based on the original work of Ref. [14] describes various features of the system in a coherent way [15-17]. The Lagrange-based (Dyson-Schwinger) approach to the problem $[3,18]$ is also making progress. One common theme to all these studies (and central to the Gribov-Zwanziger confinement scenario) is the importance of the Faddeev-Popov operator that arises after one fixes the gauge.

In addition to its noncovariant nature, Coulomb gauge is also incomplete - the gauge is only partially fixed. After applying Coulomb gauge, one can still perform time-dependent (spatially independent) gauge transformations and this raises potential questions about the strict validity of Coulomb gauge [19]. On the other hand, trying to completely fix the gauge in the continuum seems to lead to a contradiction [20]. Related to the gauge-fixing and noncovariance issues in Coulomb gauge is the problem of energy divergences. Because the gauge-fixing only involves spatial derivatives, the ghost propagator is independent of the energy and closed loops involving these ghosts appear at first sight to exhibit a pure (and unregularizable) energy divergence. It has been argued that these divergences cancel to all orders perturbatively [21]. This cancellation, which has been explicitly verified at one-loop order [22, 23], is not arbitrary: within the first order formalism, the Faddeev-Popov determinant cancels when the temporal gauge field is integrated out $[3,18]$.

In this paper, the Faddeev-Popov operator is considered with the focus on the temporal zero-modes. These modes embody both the noncovariant and incomplete aspects of the Coulomb gauge-fixing. The first order, (Lagrange-based) functional formalism in the continuum is employed. It is shown that after Gauss' law is implemented (which in the functional approach arises after integrating out the temporal gauge field), the temporal zero-modes can be accounted for and their resolution leads to a completely gauge-fixed action that is free of energy divergences (in the sense that the Faddeev-Popov determinant cancels) with a well-defined, conserved and vanishing total color charge.

\section{FUNCTIONAL FORMALISM}

The starting point for this study is the functional integral associated with continuum Yang-Mills theory (see Ref. [18] for a complete description),

$$
Z=\int \mathcal{D} \Phi \exp \left\{\imath \mathcal{S}_{Y M}\right\}, \mathcal{S}_{Y M}=\frac{1}{2} \int d^{4} x\left[E^{2}-B^{2}\right],
$$

with the chromoelectric and chromomagnetic fields given in terms of the spatial $(\vec{A})$ and temporal $(\sigma)$ gauge fields:

$$
\begin{aligned}
\vec{E}^{a} & =-\partial^{0} \vec{A}^{a}-\vec{\nabla} \sigma^{a}+g f^{a b c} \vec{A}^{b} \sigma^{c}=-\partial^{0} \vec{A}^{a}-\vec{D}^{a c}[\vec{A}] \sigma^{c}, \\
B_{i}^{a} & =\varepsilon_{i j k}\left[\nabla_{j} A_{k}^{a}-\frac{1}{2} g f^{a b c} A_{j}^{b} A_{k}^{c}\right]
\end{aligned}
$$


and where

$$
\vec{D}_{x}^{a b}[\vec{A}]=\delta^{a b} \vec{\nabla}_{x}-g f^{a c b} \vec{A}_{x}^{c}
$$

is the spatial covariant derivative. $\mathcal{D} \Phi$ denotes the functional integral measure for all field types that may be present. The action is invariant under local (and global), infinitesimal gauge transformations of the form:

$$
\begin{aligned}
& \vec{A}_{x}^{a \theta}=\vec{A}_{x}^{a}+\frac{1}{g} \vec{D}_{x}^{a b}[\vec{A}] \theta_{x}^{b}, \\
& \sigma_{x}^{a \theta}=\sigma_{x}^{a}-\frac{1}{g} \partial_{x}^{0} \theta_{x}^{a}-f^{a c b} \sigma_{x}^{c} \theta_{x}^{b} .
\end{aligned}
$$

Notice that the functional integral measure, $\mathcal{D} \Phi$, includes all those configurations related by such gauge transformations and thus contains the integration over the gauge group. Since the action is gauge invariant, integrating over the gauge group produces a (divergent) global factor which, in principle, can be absorbed into the normalization of the functional integral and in itself is harmless. However, inconsistent integration over the gauge group does become problematic when calculating gauge-variant Green's functions from the functional integral: the integration over the gauge group averages such quantities to zero. For example, in the free (non-interacting) theory it is necessary to fix the gauge in order to be able to invert the quadratic part of the action such that particle propagation may be properly defined. We fix the gauge by applying the Faddeev-Popov technique. This involves inserting the following identity $\left(\theta_{x}\right.$ is the parameter associated with the gauge transformation, see Eq. (2.4)) into the above functional integral:

$$
\mathbb{1}=\int \mathcal{D} \theta_{x} \delta(F[A]) \operatorname{det}\left[M^{b a}(y, x)\right], \quad M^{b a}(y, x)=\left.\frac{\delta F^{a}\left[A^{\theta}(x)\right]}{\delta \theta_{y}^{b}}\right|_{F=0} .
$$

We are concerned here with Coulomb gauge:

$$
F^{a}[A] \equiv \vec{\nabla} \cdot \vec{A}^{a}=0
$$

and for which the Faddeev-Popov kernel reads

$$
M^{b a}(y, x) \sim-\vec{\nabla}_{x} \cdot \vec{D}_{x}^{a b}[\vec{A}] \delta(y-x) .
$$

There are caveats to the identity, Eq. (2.5), above. When the gauge fixing is incomplete, zero-modes of the FaddeevPopov operator will arise, i.e., $\theta$ or $\vec{A}$ are such that $F\left[A^{\theta}\right]=F[A]$ and for Coulomb gauge, Eq. (2.6), there are two cases:

1. $-\vec{\nabla}_{x} \cdot \vec{D}_{x}^{a b}[\vec{A}] \theta_{x}^{b}=0$, Gribov copies for $\vec{A} \neq 0$ (generated by spatially and temporally dependent gauge transformations)

2. $-\vec{\nabla}_{x} \cdot \vec{D}_{x}^{a b}[\vec{A}] \theta^{b}(t)=0$, temporal zero-modes (generated by temporally dependent but spatially independent gauge transformations).

[We implicitly include global $(\theta=$ constant $)$ transformations into the latter category.] Clearly, in both these cases, $M$ has zero eigenvalues and consequently the Faddeev-Popov determinant also vanishes, violating Eq. (2.5) since the left-hand side cannot vanish. We must therefore modify the formalism to account for this and we write

$$
\mathbb{1}=\int \mathcal{D} \bar{\theta}_{x} \delta(F[A]) \overline{\operatorname{det}}\left[M^{b a}(y, x)\right]
$$

where $\mathcal{D} \bar{\theta}_{x}$ explicitly excludes any spatially independent $\theta(t)$ and

$$
\overline{\operatorname{det}}\left[M^{b a}(y, x)\right]=\operatorname{det}\left[M^{b a}(y, x)\right]_{-\vec{\nabla} \cdot \vec{D} \theta \neq 0}
$$

is the determinant with the zero-modes of the operator (temporal or Gribov copy) removed. We can now write our functional integral as

$$
Z=\int \mathcal{D} \Phi \delta\left(\vec{\nabla} \cdot \vec{A}^{a}\right) \overline{\operatorname{det}}[-\vec{\nabla} \cdot \vec{D}] \exp \left\{\imath \mathcal{S}_{Y M}\right\}
$$

where it is understood that the functional integration measure, $\mathcal{D} \Phi$, still contains the integration over the full gauge group. The direction(s) in group space corresponding to the zero-modes of the Faddeev-Popov kernel are still explicitly present within the functional integration and may still cause problems. 
To proceed, we convert to the first order formalism [3, 18]. We introduce an auxiliary vector field $(\vec{\pi})$ via the following identity:

$$
\exp \left\{\imath \int d^{4} x \frac{1}{2} \vec{E}^{a} \cdot \vec{E}^{a}\right\}=\int \mathcal{D} \vec{\pi} \exp \left\{\imath \int d^{4} x\left[-\frac{1}{2} \vec{\pi}^{a} \cdot \vec{\pi}^{a}-\vec{\pi}^{a} \cdot \vec{E}^{a}\right]\right\} .
$$

The $\vec{\pi}$-field is further split into components using the identity

$$
\text { const }=\int \mathcal{D} \phi \mathcal{D} \tau \exp \left\{-\imath \int d^{4} x \tau^{a}\left(\vec{\nabla} \cdot \vec{\pi}^{a}+\nabla^{2} \phi^{a}\right)\right\},
$$

changing variables $\vec{\pi}=\vec{\pi}-\vec{\nabla} \phi$ and integrating out the Lagrange multiplier field, $\tau$, to arrive at the form

$$
Z=\int \mathcal{D} \Phi \delta\left(\vec{\nabla} \cdot \vec{A}^{a}\right) \delta\left(\vec{\nabla} \cdot \vec{\pi}^{a}\right) \overline{\operatorname{det}}[-\vec{\nabla} \cdot \vec{D}] \exp \{\imath \mathcal{S}\}
$$

where

$$
\mathcal{S}=\int d^{4} x\left[-\frac{1}{2} B^{2}-\frac{1}{2} \pi^{2}-\frac{1}{2}(\nabla \phi)^{2}+\vec{\pi}^{a} \cdot \partial^{0} \vec{A}^{a}+\sigma^{a}\left(\vec{\nabla} \cdot \vec{D}^{a b} \phi^{b}+g \hat{\rho}^{a}\right)\right]
$$

and with the color charge of the gauge field

$$
\hat{\rho}^{a}=f^{a d e} \vec{A}^{d} \cdot \vec{\pi}^{e} .
$$

The advantage of the first order formalism is, of course, that the action is linear in $\sigma$ and this field can be integrated out to give

$$
Z=\int \mathcal{D} \Phi \delta\left(\vec{\nabla} \cdot \vec{A}^{a}\right) \delta\left(\vec{\nabla} \cdot \vec{\pi}^{a}\right) \overline{\operatorname{det}}[-\vec{\nabla} \cdot \vec{D}] \delta\left(\vec{\nabla} \cdot \vec{D}^{a b} \phi^{b}+g \hat{\rho}^{a}\right) \exp \left\{\imath \mathcal{S}^{\prime}\right\}
$$

where

$$
\mathcal{S}^{\prime}=\int d^{4} x\left[-\frac{1}{2} B^{2}-\frac{1}{2} \pi^{2}-\frac{1}{2}(\nabla \phi)^{2}+\vec{\pi}^{a} \cdot \partial^{0} \vec{A}^{a}\right] .
$$

The $\delta$-functional constraint on the scalar field $\phi$ is the functional expression of Gauss' law. To resolve the constraint and eliminate the functional integration over the scalar field $\phi$, we first must investigate the Faddeev-Popov operator a little more closely. The eigenvalue equation for the operator is

$$
-\vec{\nabla} \cdot \vec{D}_{x}^{a b}[\vec{A}] \varphi_{n}^{b}(\vec{x} ; t)=\lambda_{n} \varphi_{n}^{a}(\vec{x} ; t)
$$

with a complete orthonormal basis $\varphi_{n}^{a}(\vec{x} ; t)$ satisfying

$$
\int d^{4} x \varphi_{m}^{* a}(\vec{x} ; t) \varphi_{n}^{a}(\vec{x}, t)=\delta_{m n} .
$$

However, since the time dependence of the operator is only implicit (within the spacetime dependent field $\vec{A}_{x}$ ), the time argument is only a label (in the sense that there is a different spatial operator at each time). We could state that the eigenvalue equation is evaluated at a specific time and for which the eigenfunctions are spatially orthonormalized at this time. In this case, the eigenvalues become implicitly time dependent in that they refer to the eigenvalue equation at a particular time, $t$ :

$$
-\vec{\nabla} \cdot \vec{D}_{x}^{a b}[\vec{A}] \varphi_{t n}^{b}(\vec{x})=\lambda_{t n} \varphi_{t n}^{a}(\vec{x}), \quad \int d^{3} x \varphi_{t m}^{* a}(\vec{x}) \varphi_{t n}^{a}(\vec{x})=\delta_{m n}
$$

We use the convention that $\lambda_{t n=0}=0$ denotes the collection of zero modes of the Faddeev-Popov operator. The temporal zero-modes are spatially constant and for $S U\left(N_{c}\right)$, there are $N_{c}^{2}-1$ such linearly independent eigenvectors in the color space which when necessary, we label by an additional index $\mu: \varphi_{t 0}^{b}(\vec{x})=\varphi_{t 0 \mu}^{b}$ (the adjoint color index, $b$ here, labels the component of the $\mu$-th vector). Using the complete orthonormal basis, Eq. (2.20), we can expand the field, $\phi$, and color charge, $\hat{\rho}$, as series (index $\mu$ is implicit within the zero modes):

$$
\phi_{x}^{a}=\sum_{n=0}^{\infty} b_{n}(t) \varphi_{t n}^{a}(\vec{x}), \quad \hat{\rho}_{x}^{a}=\sum_{n=0}^{\infty} a_{n}(t) \varphi_{t n}^{a}(\vec{x})
$$


where the coefficients are time dependent and, in particular,

$$
a_{0 \mu}(t) \sim \int d^{3} x \varphi_{t 0 \mu}^{* a}(\vec{x}) \hat{\rho}_{x}^{a} .
$$

Having identified the zero-modes, albeit formally, we may define the inverse Faddeev-Popov operator in their absence, $\bar{M}$ :

$$
[-\vec{\nabla} \cdot \vec{D}] \bar{M} \Psi_{x}=\bar{M}[-\vec{\nabla} \cdot \vec{D}] \Psi_{x}=\Psi_{x}
$$

such that

$$
\operatorname{det} \bar{M}=\prod_{n \neq 0} \lambda_{t n}^{-1}=\overline{\operatorname{det}}[-\vec{\nabla} \cdot \vec{D}]^{-1}
$$

Returning to the functional integral over $\phi$ within the expression, Eq. (2.16), we may now write

$$
\begin{aligned}
\int & \mathcal{D} \phi \delta\left(\vec{\nabla} \cdot \vec{D}^{a b} \phi^{b}+g \hat{\rho}^{a}\right) \exp \left\{\imath \int d^{4} x\left[-\frac{1}{2}(\nabla \phi)^{2}\right]\right\} \\
= & \int\left[\prod_{n=0}^{\infty} d b_{n}(t) \delta\left(-b_{n}(t) \lambda_{t n}+g a_{n}(t)\right)\right] \exp \left\{\imath \int d^{4} x \frac{1}{2} \sum_{m, n=0}^{\infty} b_{m}^{*}(t) b_{n}(t) \varphi_{t m}^{* a}(\vec{x}) \nabla_{x}^{2} \varphi_{t n}^{a}(\vec{x})\right\} \\
= & \prod_{\mu} \delta\left(g a_{0 \mu}(t)\right) \int_{-\infty}^{\infty} d b_{0 \mu}(t) \int\left[\prod_{n=1}^{\infty} d b_{n}(t) \delta\left(-b_{n}(t) \lambda_{t n}+g a_{n}(t)\right)\right] \times \\
& \exp \left\{\frac { \imath } { 2 } \int d ^ { 4 } x \left[\left|b_{0 \mu}(t)\right|^{2} \varphi_{t 0 \mu}^{* a}(\vec{x}) \nabla_{x}^{2} \varphi_{t 0 \mu}^{a}(\vec{x})+2 \sum_{n=1}^{\infty} b_{0 \mu}^{*}(t) b_{n}(t) \varphi_{t 0 \mu}^{* a}(\vec{x}) \nabla_{x}^{2} \varphi_{t n}^{a}(\vec{x})\right.\right. \\
& \left.\left.+\sum_{m, n=1}^{\infty} b_{m}^{*}(t) b_{n}(t) \varphi_{t m}^{* a}(\vec{x}) \nabla_{x}^{2} \varphi_{t n}^{a}(\vec{x})\right]\right\} \\
= & \prod_{\mu} \delta\left(g a_{0 \mu}(t)\right) \frac{\operatorname{det}[-\vec{\nabla} \cdot \vec{D}]^{-1} \int_{-\infty}^{\infty} d b_{0 \mu}(t) \times}{} \\
& \quad \exp \left\{\frac { \imath } { 2 } \int d ^ { 4 } x \left[\left|b_{0 \mu}(t)\right|^{2} \varphi_{t 0 \mu}^{* a}(\vec{x}) \nabla_{x}^{2} \varphi_{t 0 \mu}^{a}(\vec{x})+2 g \sum_{n=1}^{\infty} b_{0 \mu}^{*}(t) \frac{a_{n}(t)}{\lambda_{t n}} \varphi_{t 0 \mu}^{* a}(\vec{x}) \nabla_{x}^{2} \varphi_{t n}^{a}(\vec{x})\right.\right. \\
& \left.\left.+g^{2} \sum_{m, n=1}^{\infty} \frac{a_{m}^{*}(t) a_{n}(t)}{\lambda_{t m}^{*} \lambda_{t n}} \varphi_{t m}^{* a}(\vec{x}) \nabla_{x}^{2} \varphi_{t n}^{a}(\vec{x})\right]\right\} .
\end{aligned}
$$

If we neglect the (spatially dependent) Gribov copies, the temporal zero modes can be completely eliminated by noting that $\vec{\nabla}_{x} \varphi_{t 0 \mu}^{a}=0$, such that the integrals over the $b_{0 \mu}(t)$ are overall (divergent) constants which can be absorbed into the normalization [26]. Thus, using Eq. (2.22)

$$
\begin{aligned}
& \int \mathcal{D} \phi \delta\left(\vec{\nabla} \cdot \vec{D}^{a b} \phi^{b}+g \hat{\rho}^{a}\right) \exp \left\{\imath \int d^{4} x\left[-\frac{1}{2}(\nabla \phi)^{2}\right]\right\} \\
& =\prod_{\mu} \delta\left(g \varphi_{t 0 \mu}^{* a} \int d^{3} x \hat{\rho}_{x}^{a}\right) \overline{\operatorname{det}}[-\vec{\nabla} \cdot \vec{D}]^{-1} \exp \left\{\frac{\imath}{2} \int d^{4} x\left[g^{2} \sum_{m, n=1}^{\infty} \frac{a_{m}^{*}(t) a_{n}(t)}{\lambda_{t m}^{*} \lambda_{t n}} \varphi_{t m}^{* a}(\vec{x}) \nabla_{x}^{2} \varphi_{t n}^{a}(\vec{x})\right]\right\}
\end{aligned}
$$

Now, because the temporal zero-mode is also a zero-mode of the Laplacian, we can infer that the ratio $\vec{\nabla} \varphi_{t 0 \mu} / \lambda_{t 0}$ is finite and that if we multiply by $a_{0 \mu}(t)=0$ then the product vanishes. Thus, the sum in the exponential can be extended to include the zero-modes without reintroducing any ambiguities (we are, after all, only adding zero) and we have the form

$$
\begin{aligned}
& \int \mathcal{D} \phi \delta\left(\vec{\nabla} \cdot \vec{D}^{a b} \phi^{b}+g \hat{\rho}^{a}\right) \exp \left\{\imath \int d^{4} x\left[-\frac{1}{2}(\nabla \phi)^{2}\right]\right\} \\
& =\prod_{\mu} \delta\left(g \varphi_{t 0 \mu}^{* a} \int d^{3} x \hat{\rho}_{x}^{a}\right) \overrightarrow{\operatorname{det}}[-\vec{\nabla} \cdot \vec{D}]^{-1} \exp \left\{\frac{\imath}{2} \int d^{4} x\left[g^{2} \hat{\rho_{x}^{a}}\left[-\vec{\nabla}_{x} \cdot D_{x}^{a b}[\overrightarrow{A]}]\right]^{-1} \nabla^{2}\left[-\vec{\nabla}_{x} \cdot D_{x}^{b c}[\vec{A}]\right]^{-1} \hat{\rho_{x}^{c}}\right]\right\}
\end{aligned}
$$


where the quantities in the exponent formally include the zero-modes. As demonstrated, these zero-modes play no role other than to allow us to write the action conventionally in terms of the full fields; however, nontrivially, these full fields have been shown to be well-defined insofar as the temporal zero-modes are concerned. Since the $\varphi_{t 0 \mu}^{a}$ are $\left(N_{c}^{2}-1\right)$ linearly independent vectors in the adjoint color space, we also have that

$$
\prod_{\mu} \delta\left(g \varphi_{t 0 \mu}^{* a} \int d^{3} x \hat{\rho}_{x}^{a}\right) \rightarrow \prod_{a} \delta\left(\int d^{3} x \hat{\rho}_{x}^{a}\right) .
$$

Returning to the original functional integral, Eq. (2.16), and substituting in the above result for the $\phi$ integration, Eq. (2.27), we see immediately that the modified Faddeev-Popov determinants cancel, leaving

$$
Z=\int \mathcal{D} \Phi \delta\left(\vec{\nabla} \cdot \vec{A}^{a}\right) \delta\left(\vec{\nabla} \cdot \vec{\pi}^{a}\right) \delta\left(\int d^{3} x \hat{\rho}_{x}^{a}\right) \exp \left\{\imath \mathcal{S}^{\prime \prime}\right\}
$$

with

$$
\mathcal{S}^{\prime \prime}=\int d^{4} x\left[-\frac{1}{2} B^{2}-\frac{1}{2} \pi^{2}+\vec{\pi}^{a} \cdot \partial^{0} \vec{A}^{a}+\frac{1}{2} g^{2} \hat{\rho_{x}^{a}}\left[-\vec{\nabla}_{x} \cdot D_{x}^{a b}[\vec{A}]\right]^{-1} \nabla^{2}\left[-\vec{\nabla}_{x} \cdot D_{x}^{b c}[\vec{A}]\right]^{-1} \hat{\rho_{x}^{c}}\right] .
$$

Given the definition of the color charge $\hat{\rho}^{a}$, Eq. (2.15), it is clear that the argument of its $\delta$-functional constraint $\left(\int d^{3} x f^{a b c} \vec{A}^{b} \cdot \vec{\pi}^{c}\right)$ must vanish at each time, $t$. Further, the vanishing of the charge is invariant under temporal gauge transformations since (using the fundamental representation for the colored fields and denoting the spatially independent gauge transformation in this representation $U_{t}$ )

$$
0=\int d^{3} x \rho=\int d^{3} x[\vec{A}, \vec{\pi}] \rightarrow \int d^{3} x\left[U_{t} \vec{A} U_{t}^{\dagger}, U_{t} \vec{\pi} U_{t}^{\dagger}\right]=U_{t} \int d^{3} x[\vec{A}, \vec{\pi}] U_{t}^{\dagger}=0 .
$$

The action, $\mathcal{S}^{\prime \prime}$ given by Eq. (2.30), is however no longer invariant under such temporal transformations (courtesy of the $\vec{\pi}^{a} \cdot \partial^{0} \vec{A}^{a}$ term which remains after we have integrated out the $\sigma$-field) and the temporal zero-modes of our new functional integral, Eq. (2.29), do not exist. This functional integral is thus fully gauge-fixed (it contains no more zero-modes, except those Gribov copies that we ignore here) and constrains the conserved, total color charge to be vanishing:

$$
\int d^{3} x \hat{\rho}_{x}^{a}=\int d^{3} x f^{a b c} \vec{A}^{b} \cdot \vec{\pi}^{c}=0
$$

The appearance of the $\delta$-functional constraint in Eq. (2.29), which ensures the vanishing of the total color charge is the main result of this work. Given that the action, Eq. (2.30), is unchanged from the form originally derived in [3], the consideration of the zero-modes leads to the further observation that the dynamics of the theory are unaltered.

If quarks were to be included in the original action, then the color charge acquires an extra component:

$$
\hat{\rho}_{q}^{a}=\bar{q} T^{a} \gamma^{0} q
$$

where $T^{a}$ is the (Hermitian) generator of the gauge group, $q$ is the quark field and $\bar{q}$ its conjugate. The total charge is constrained as before, i.e.,

$$
\int d^{3} x\left[\hat{\rho}^{a}+\hat{\rho}_{q}^{a}\right]=0
$$

The inclusion of quarks into the first order formalism is currently being studied [24].

\section{SUMMARY AND CONCLUSIONS}

The temporal (and global) zero-modes of the Faddeev-Popov operator for Coulomb gauge, continuum Yang-Mills theory within the first order formalism have been studied. The explicit separation of such modes leads to a well-defined total color charge that is conserved and vanishing at all times.

The appearance of the total color charge is interesting for several reasons. Firstly, that the total charge of the system is conserved and vanishing is a necessary condition for confinement - one could not expect only color singlet hadrons to emerge if the system were not so. As such, the properties of this charge may be regarded as a proof that 
at the least, the total system (i.e., the universe) is colorless. This does not however say much about the confinement of quarks and gluons, or the observed spectrum of hadrons. That being said though, whilst the temporal zero-modes lead only to a total conserved color charge, one may speculate about the role of the Gribov copies (i.e., the spatially dependent zero-modes) in confinement. This is the premise of the Gribov-Zwanziger confinement scenario [1-3] and the above analysis intuitively supports this.

Second, given that the temporal zero-modes drop out of the functional integral without modification of the effective, gauge-fixed action despite the fact that the (Coulomb) gauge-fixing is incomplete leads to the conclusion that no further gauge-fixing considerations are necessary and that Coulomb gauge is a well-defined choice of gauge insofar as the dynamics are concerned (leaving aside the definition of the physical state space). Further, since such temporal zero-modes are associated with the ghost energy divergence problem it is clear that these divergences must cancel in final (physically meaningful) expressions, at least in principle (although they may occur in individual components).

Thirdly, Gauss' law (which here appears as the $\delta$-functional constraint on the auxiliary $\phi$-field in Eq. (2.16) and arises when one integrates out the temporal, $\sigma$-field) plays a pivotal role in the extraction of the zero-modes and therefore in both the definition (and conservation) of the total color charge and in resolving the incompleteness of the gauge (see the previous paragraph). This is not surprising, since Gauss' law defines the charge via the generator of gauge transformations [16]. In the Hamiltonian approach to Yang-Mills theory [14], the imposition of Gauss' law as a constraint on the physical state space ensures gauge invariance in exactly this way. The resolution of Gauss' law in Coulomb gauge results in the explicit appearance of the nonabelian Coulomb potential in the Hamiltonian (and also here as the last term in the effective action, Eq. (2.30)).

Fourthly, whilst the first order formalism has been employed here, the manipulations required to construct this formalism from the more usual second order formalism comprise identities that merely serve to linearize the action with respect to the temporal gauge field, $\sigma$. Thus, what is true in the first order formalism will also be true in the second order formalism, although it will be manifested differently. The eventual form of the functional integral, Eq. (2.29), with the effective action, Eq. (2.30), here are non-local expressions and as such are not amenable to renormalization (that the first order formalism is not multiplicatively renormalizable is known [3]). In the standard, local version of the second order formalism (i.e., where the Faddeev-Popov determinant is expressed in terms of Grassmannian ghost fields etc.) which appears to be locally renormalizable (although there is as yet no conclusive proof) and therefore more suited to calculation, it may indeed not be possible to write the constraint that the total color charge is conserved and vanishing in convenient form but this does not mean that this is not true - quite the opposite. In any event, the dynamics of the theory are unaltered and in particular from a practical standpoint, the incomplete gauge-fixing poses no fundamental problem and the ghost energy divergences will cancel in the second order formalism just as in the first.

It is worth pointing out that in principle, the $\vec{\pi}$-field in the final expressions, Eq. (2.29) and Eq. (2.30), can be integrated out (the exponent is at most quadratic in $\vec{\pi}$ ) and the $\delta$-functional constraint on the total charge explicitly resolved. Whether or not the resulting expressions have any practical use however, remains to be seen, because of their highly non-local nature.

Finally, the gauge invariance of the theory as applied to the Green's functions leads to Ward-Takahashi and SlavnovTaylor identities. If one naively applies a temporal gauge transformation and tries to construct these identities, the resulting expressions are meaningless since the functional integration measure is not properly defined. As such, this is the only visible effect of leaving the temporal zero-modes of the Faddeev-Popov operator in the functional integral. However, once recognized this poses no problem in practice since to derive such identities one generally considers a fully spacetime dependent gauge transformation. The derivation of such identities is the focus of present work [25].

\section{Acknowledgments}

This work has been supported by the Deutsche Forschungsgemeinschaft (DFG) under contracts no. DFG-Re856/6-1 and DFG-Re856/6-2.

[1] V. N. Gribov, Nucl. Phys. B 139 (1978) 1.

[2] D. Zwanziger, Nucl. Phys. B 485, 185 (1997) [arXiv:hep-th/9603203].

[3] D. Zwanziger, Nucl. Phys. B 518 (1998) 237.

[4] G. Burgio, M. Quandt and H. Reinhardt, arXiv:0807.3291 [hep-lat].

[5] A. Cucchieri and D. Zwanziger, Phys. Rev. D 65, 014001 (2002) [arXiv:hep-lat/0008026].

[6] K. Langfeld and L. Moyaerts, Phys. Rev. D 70, 074507 (2004) [arXiv:hep-lat/0406024].

[7] M. Quandt, G. Burgio, S. Chimchinda and H. Reinhardt, PoS LAT2007, 325 (2007) [arXiv:0710.0549 [hep-lat]]. 
[8] A. Voigt, E. M. Ilgenfritz, M. Muller-Preussker and A. Sternbeck, PoS LAT2007, 338 (2007) [arXiv:0709.4585 [hep-lat]]. [9] A. Cucchieri, AIP Conf. Proc. 892, 22 (2007) [arXiv:hep-lat/0612004].

[10] A. P. Szczepaniak and E. S. Swanson, Phys. Rev. D 65 (2002) 025012 [arXiv:hep-ph/0107078].

[11] A. P. Szczepaniak, Phys. Rev. D 69 (2004) 074031 [arXiv:hep-ph/0306030].

[12] C. Feuchter and H. Reinhardt, Phys. Rev. D 70 (2004) 105021 [arXiv:hep-th/0408236]; C. Feuchter and H. Reinhardt, arXiv:hep-th/0402106.

[13] H. Reinhardt and C. Feuchter, Phys. Rev. D 71 (2005) 105002 [arXiv:hep-th/0408237].

[14] N. H. Christ and T. D. Lee, Phys. Rev. D 22, 939 (1980) [Phys. Scripta 23, 970 (1981)].

[15] H. Reinhardt and D. Epple, Phys. Rev. D 76, 065015 (2007) [arXiv:0706.0175 [hep-th]].

[16] H. Reinhardt, arXiv:0803.0504 [hep-th].

[17] D. R. Campagnari and H. Reinhardt, arXiv:0807.1195 [hep-th].

[18] P. Watson and H. Reinhardt, Phys. Rev. D 75, 045021 (2007) [arXiv:hep-th/0612114].

[19] L. Baulieu and D. Zwanziger, Nucl. Phys. B 548, 527 (1999) [arXiv:hep-th/9807024].

[20] P. Watson and H. Reinhardt, arXiv:0711.2997 [hep-th].

[21] A. Niegawa, M. Inui and H. Kohyama, Phys. Rev. D 74, 105016 (2006) [arXiv:hep-th/0607207].

[22] P. Watson and H. Reinhardt, Phys. Rev. D 76, 125016 (2007) [arXiv:0709.0140 [hep-th]].

[23] P. Watson and H. Reinhardt, Phys. Rev. D 77, 025030 (2008) [arXiv:0709.3963 [hep-th]].

[24] C. Popovici, P. Watson and H. Reinhardt, in preparation.

[25] P. Watson and H. Reinhardt, in preparation.

[26] One can appreciate the nature of the full Gribov problem very clearly within this context. The eigenfunctions, $\varphi_{t n}^{a}(\vec{x})$, of the Faddeev-Popov operator in this case are implicitly dependent on the field configurations $\vec{A}(x)$ which, in the complete functional integral, are being integrated over. Without the simplification as in the temporal case considered in this study, one is left with a seemingly intractable problem. 